\section{A unique case of hemi-tongue pseudohypertrophy, necrotizing myopathy, and erythema nodosum}

\author{
Kota Sato, Yoshiaki Takahashi, \\ Toru Yamashita, Mami Takemoto, \\ Nozomi Hishikawa, Shang Jinwei, \\ Yasuyuki Ohta, Koji Abe \\ Department of Neurology, Graduate \\ School of Medicine, Dentistry \\ and Pharmaceutical Sciences, \\ Okayama University, Japan
}

\begin{abstract}
A 46-year-old woman developed slowly progressive tongue weakness with a pseudohypertrophic change on the right side of her tongue. She subsequently developed weakness in her proximal lower extremities, skin erythema and a sustained increase of muscle enzymes at $11 \mathrm{M}$ after the onset. A biopsy of the quadriceps muscle showed necrotizing myopathy and a skin biopsy showed erythema nodosum. The present case showed characteristic clinical manifestations that may represent a rare variant of sarcoidosis.
\end{abstract}

\section{Introduction}

Herein, we report a 46-year-old woman who developed slowly progressive tongue weakness with a pseudohypertrophic change, and consecutively displayed an increase in muscle enzymes, weakness in her proximal lower extremities, and skin erythema at $11 \mathrm{M}$ after the onset.

\section{Case Report}

A woman began to feel a swelling of the tongue at the age of 46 . Within 3 months (M), she had developed dysarthria, and dysphasia by $4 \mathrm{M}$, and because her symptoms gradually worsened, she was admitted to our hospital by $6 \mathrm{M}$.

Upon initial admission to our hospital, the right side of her tongue was mildly swollen, and upward tongue movement was primarily disturbed (Figure 1A-D). She showed mild weakness in her neck flexion (MMT 4), but did not show any other neurological deficits. Laboratory data revealed a normal ESR (11/24 mm) and WBC (4,620 $/ \mu \mathrm{L})$, but serum $\mathrm{CK}$ was markedly elevated at 2,940 IU/L with a mild increase in aldolase (ALD, 23.1 U/L), AST (68 IU/L), ALT (72 IU/L) and LDH (696 U/L). Serum calcium $(9.1 \mathrm{mg} / \mathrm{dL})$, angiotensin converting enzyme (ACE, 7.0 U/L), and soluble IL2 receptor (sIL-2R, $417 \mathrm{U} / \mathrm{mL}$ ) were normal. Serum autoantibodies such as ANA, SS-A, SS-B, ds-DNA antibody, MPOANCA, and PR3-ANCA were all negative. The thyroid hormone was normal. Several infection tests for syphilis, hepatitis B, hepatitis $\mathrm{C}$ and HIV all tested negative. Analysis of cerebrospinal fluid (CSF) revealed a normal cell count $(1 / \mu \mathrm{L})$, protein $(38 \mathrm{mg} / \mathrm{dL})$.

A whole-body CT showed neither lymphadenopathy (BHL) nor tumors. A brain MRI showed no lesion in the medulla oblongata or hypoglossal canal (Figure 1E). A tongue MRI showed hyperintense on the right side of her tongue, both in the $\mathrm{T} 1$ weighted image (T1WI) and the T2 weighted image (T2WI) (Figure 1F and G, arrowheads), which was suppressed in fat-suppression T1WI and was not enhanced by gadolinium (Gd) (Figure 1H, arrowhead). A tongue biopsy of the right side after $7 \mathrm{M}$ showed that it was edematous and fibrotic without any striated muscles (Figure 1I) but without any invasion of malignant cells, infiltration of inflammatory cells, or granuloma. These findings indicate that the tongue lesion was not truly hypertrophic but was instead pseudohypertrophic. The patient was tentatively diagnosed as having idiopathic unilateral hypoglossal nerve palsy, and was discharged after $7 \mathrm{M}$.

After discharge, she gradually felt weakness in her legs when climbing stairs. Upon a second admission at $11 \mathrm{M}$ after the onset, a newly discovered skin erythema was observed on her left foot (Figure 1J, arrowhead). She also showed bilateral iliopsoas muscle weakness (MMT 4) without myalgia. Laboratory data revealed similar results as her first admission such as a normal ESR $(14 / 31 \mathrm{~mm})$, WBC $(4,210 / \mu \mathrm{L})$, CRP $(0.04 \mathrm{mg} / \mathrm{dL})$, but increase in serum CK (2,428 IU/L), ALD (26.0 U/L), AST (50 IU/L), ALT (45 IU/L), and LDH (616 IU/L). Serum lysozyme $(4.7 \mu \mathrm{g} / \mathrm{mL})$, SRP antibody, and HMGCR antibody were negative. The HLA-A haplotype was A24/A33, and the HLA-B was B44/B52. ${ }^{67} \mathrm{Ga}$ scintigraphy revealed no accumulation in the entire body. Needle electromyography showed no evident myogenic or neurogenic changes in her extremities.

A skin biopsy of the foot erythema (Figure 1J) showed mild lymphoid cell infiltration between subcutaneous adipose tissue indicating a lobular panniculitis (Figure 1K,L; arrowheads). A thigh MRI revealed a hyperintense lesion in the distal
Correspondence: Koji Abe, Department of Neurology, Okayama University Graduate School of Medicine, Dentistry and Pharmacy, 2-5-1 Shikata-cho, Okayama 700-8558, Japan. Tel.: +81.86.2357365 - Fax: +81.86.2357368. E-mail: kosatou@cc.okayama-u.ac.jp

Key words: tongue pseudohypertrophy, hypoglossal nerve palsy, necrotizing myopathy, erythema nodosum, sarcoidosis.

Contributions: the authors contributed equally.

Conflict of interest: the authors declare no potential conflict of interest.

Funding: none.

Received for publication: 27 August 2018. Accepted for publication: 31 August 2018.

This work is licensed under a Creative Commons Attribution NonCommercial 4.0 License (CC BY-NC 4.0).

(C) Copyright K. Sato et al., 2018

Licensee PAGEPress, Italy

Neurology International 2018; 10:7852

doi:10.4081/ni.2018.7852

portion of the right quadriceps muscle on T2WI and fat-suppression T2WI (Figure $1 \mathrm{M}, \mathrm{N}$; arrowheads). A muscle biopsy of the right quadriceps showed mild variation in fiber size (Figure 10) with necrotic fibers (Figure 1O-Q, arrowheads). Since her muscle weakness was moderately relieved without any treatment, she was discharged $13 \mathrm{M}$ after the onset and her conditions are being carefully followed up.

\section{Discussion and Conclusions}

The present case initially showed weakness on the right side of the tongue with pseudohypertrophy (Figure 1A-D) and fatty degeneration (Figure 1F-H). Tongue weakness and atrophy are usually caused by hypoglossal nerve palsy, but tongue pseudohypertrophy is also caused by idiopathic hypoglossal nerve palsy. ${ }^{1}$ As for the present case, a tongue MRI and tongue biopsy showed similar finding to the previous case. ${ }^{1}$ In addition, a unilateral limited tongue lesion suggests hypoglossal nerve palsy (Figure 1F-H).

Lobular panniculitis of skin erythema is a typical pathological change to erythema nodosum, which is an important physical finding in systemic diseases such as sarcoidosis, Behçet's disease, infection, and cancer. ${ }^{2}$ In the present case, both infection and malignancy were ruled out by her clin- 

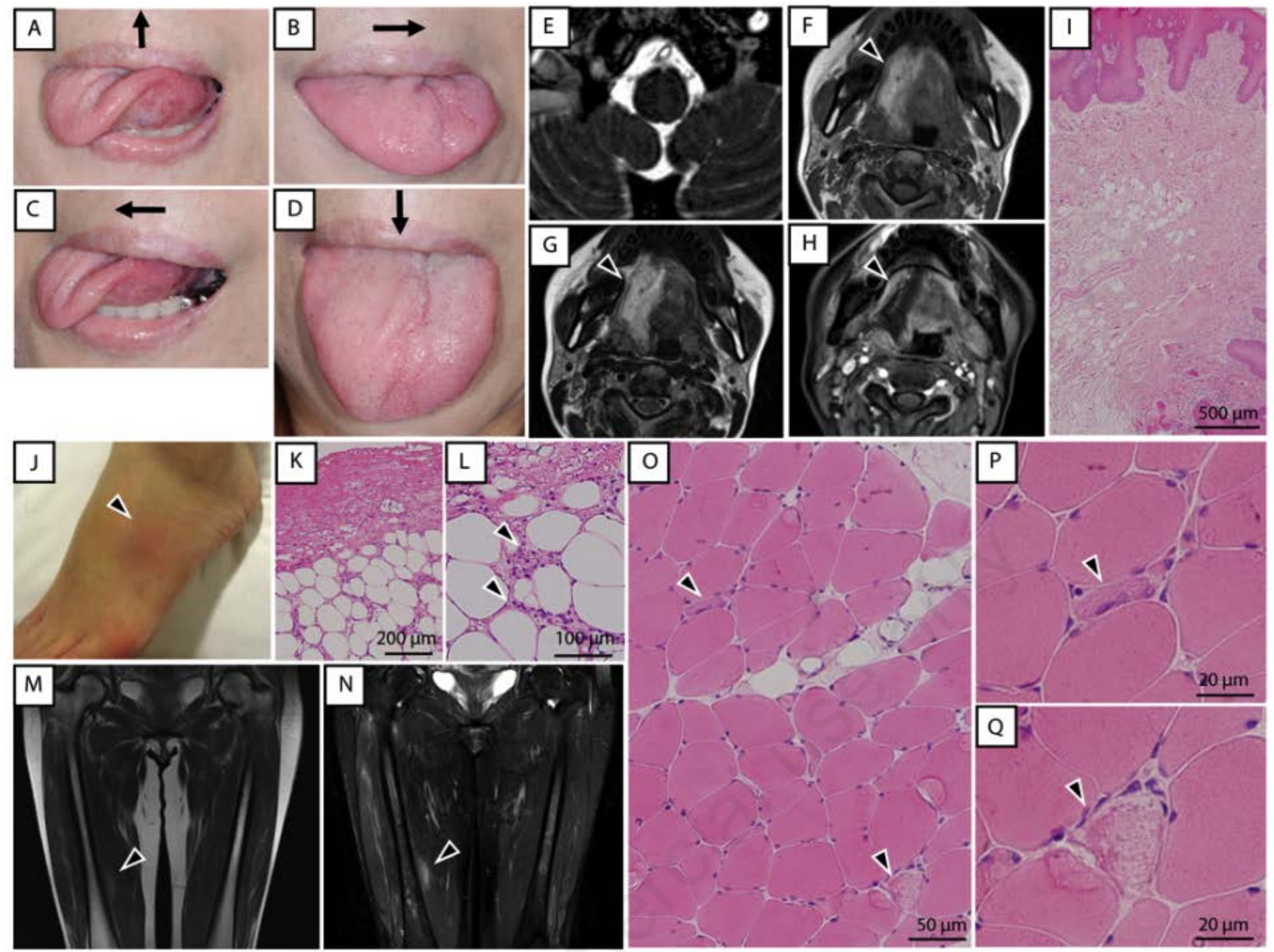

Figure 1. Tongue movements in four directions, upward (A), leftward (B), rightward (C), and downward (D), showing disturbed upward tongue movement $(A)$ and swelling on the right side of the tongue (D). Head MRI (E-H) showing no lesion in medulla oblongata and hypoglossal nerves on T2WI (E), but hyperintensive right side of the tongue on T1WI (F) and T2WI (G) (arrowheads), and a decreased intensity on fat-suppression T1WI without Gd enhancement $(\mathrm{H})$. (I) Right tongue biopsy showing it to be edematous and fibrotic without any striated muscles. Erythema on the left foot (J, arrowhead) and its biopsy (K, L), showing lobular panniculitis (L, arrowheads). Thigh MRI on T2WI (M) and fat-suppression T2WI (N) showing hyperintense lesion in the distal portion of the right quadriceps femoralis (arrows). (O-Q) Quadriceps muscle biopsy showing mild variation in fiber size $(\mathrm{O})$ with necrotic fibers $(\mathrm{O}-\mathrm{Q}$, arrowheads).

ical course, blood tests, and whole-body CT. A quadriceps muscle biopsy revealed mild necrotizing myopathy (Figure 1O-P; arrows). Sarcoidosis can present both myopathy without typical lymphadenopathy, ${ }^{3}$ and hypoglossal nerve palsy. 4,5 Although sarcoidosis-specific findings such as elevated serum marker (ACE, lysosome, and $\mathrm{Ca}$ ), lymphadenopathy, the accumulation of ${ }^{67} \mathrm{Ga}$, and noncaseating granuloma were not shown in the present case, sarcoidosis is more likely than other etiologies. A successive and careful follow-up will hopefully uncover the exact diagnosis in the future.

\section{References}

1. Holle D, Kastrup O, Sheu S, et al. Neurological picture. Tongue pseudohypertrophy in idiopathic hypoglossal nerve palsy. JNNP 2009;80:1393.

2. Schwartz R, Nervi S. Erythema nodosum: a sign of systemic disease.
Am Fam Physician 2007;75:695-700.

3. Berger C, Sommer C, Meinck H. Isolated sarcoid myopathy. Muscle Nerve 2002;26:553-6.

4. Pawate S, Moses S, Sriram S. Presentations and outcomes of neurosarcoidosis: a study of 54 cases. QJM 2009; 102:449-60.

5. Sharma B. Dubey P, Kumar S, et al. Isolated unilateral hypoglossal nerve palsy: a study of 12 cases. J Neurol Neurosci 2010;2:1-5. 